\title{
Measurement of the Earth's Magnetic Field Vector Based on Zero Field Finding Algorithm Using Optically Pumped Magnetometers
}

\author{
Amin Zamani ${ }^{\mathrm{a}}$, Maliheh Ranjbaran ${ }^{\mathrm{a}}$, Mohammad Mehdi Tehranchi ${ }^{\mathrm{a}, \mathrm{b}, *}$, Seyed Mohammad \\ Hosein Khalkhali ${ }^{c}$, Seyedeh Mehri Hamidi ${ }^{a}$ \\ ${ }^{a}$ Laser and Plasma Research Institute, Shahid Beheshti University, Tehran, Iran \\ ${ }^{b}$ hysics Department, Shahid Beheshti University, Tehran, Iran \\ ${ }^{\text {c}}$ Physics Department, Kharazmi University, Tehran, Iran
}

Corresponding author Email: teranchi@ sbu.ac.ir

\begin{abstract}
Regular paper: Received: Jun. 18, 2019, Revised: Dec. 11, 2019, Accepted: Dec. 13, 2019, Available Online: June. 30, 2020, DOI: 10.29252/ijop.14.1.25
\end{abstract}

\begin{abstract}
Atomic magnetometers have found widespread applications in precise measurement of the Earth's magnetic field due to their high sensitivity. In these measurements, various methods have been utilized to compensate the Earth's magnetic field in an unshielded environment. In this paper, we have proposed a method based on finding the minimum resonance frequency (corresponding to minimum magnetic field) by producing the opposite magnetic field through three pairs of Helmholtz coils. The exact value of the Earth's magnetic field vector is obtained as $35.132 \mu \mathrm{T}$ with an accuracy of $2 \mathrm{nT}$ by using this method.
\end{abstract}

KEYWORDS: Atomic magnetometers, Earth magnetic field, Helmholtz coils, Zeeman Effect.

\section{I.INTRODUCTION}

The Earth's magnetic field is one of the most important causes of life's existence on the planet. High sensitivity measurement of this magnetic field and its variations has recently attracted considerable attention in various applications such as geophysical exploration and study of mineral deposits [1]-[2], flight navigation [3]-[4], and also geothermal energy monitoring [5]. In these precise measurements, fluxgate magnetometers, proton precession magnetometers [6], superconducting quantum interference devices [7] and recently, optically pumped magnetometers (OPMs) [8]-[9] have been utilized. Intrinsic advantages of the OPMs, such as non-cryogenic operation, small size, and low cost have particularly made them one of the best candidates for high-sensitivity magnetometry.

OPMs are based on the measurement of Larmor precession frequency of spin-polarized alkali atoms in the presence of a magnetic field. The spin polarization is produced through optical pumping process. This process creates population imbalance in the magnetic sublevels by transferring angular momentum to the atoms from a resonant circularly polarized laser light. The laser light is not absorbed while the atoms are pumped into the highest sublevel of the ground state (dark state). A transition between neighboring magnetic sublevels (synchronization of the spin precession) occurs by using a weak oscillating radio frequency (RF) magnetic field. The RF magnetic field causes resonant depolarization when its frequency coincides with the Larmor precession frequency. The Larmor frequency, related to the strength of the magnetic field, can be detected by investigating the modification of optical absorptive (quadrature) component and dispersive (in-phase) component of the resonance signal [10]. 
The highly sensitive OPMs, were developed to measure the geomagnetic fields in unshielded environments [11], by utilizing compensation coils [9] and field modulations techniques [12]. In this paper, we have proposed a novel technique based on zero field finding algorithm. In this algorithm the minimum resonance frequency associated with the minimum magnetic field is derived in three dimensions in two steps: approximate compensation and accurate compensation of the Earth's field. In this way, the Earth's magnetic field components are measured and applied oppositely. This makes it possible for the atomic magnetometers to work in an unshielded environment.

\section{EXPERIMENTAL SETUP}

The experimental setup is shown in Fig. 1. The heart of the magnetometer is a cylindrical cell containing rubidium atoms with a length of $50 \mathrm{~mm}$ and diameter of $25 \mathrm{~mm}$. The cell contains both isotopes of rubidium $\left({ }^{85} \mathrm{Rb}\right.$ and ${ }^{87} \mathrm{Rb}$ ) with natural abundances and 10 torr of $\mathrm{N}_{2}$ as a quenching gas. The inner surface of the cell was coated by octadecyltrichlorosilane
(OTS) to reduce spin destruction from wall collisions. To vaporize the rubidium, the cell was heated to $\sim 55^{\circ} \mathrm{C}$ with a resistive heater wrapped around it, and the temperature was monitored and controlled via a PID temperature controller.

A distributed feedback (DFB) diode laser tuned to $\mathrm{D}_{1}$ resonance of $\mathrm{Rb}$ atoms at $794.8 \mathrm{~nm}$ was used for optical pumping along the cell axis. The laser beam was transported by a multi-mode fiber to the magnetometer, where it was collimated and circularly polarized before entering the sensor cell. The beam pumped the atoms to the highest magnetic sublevels of the ground state. The atoms transferred to nearest spin states by using a radio frequency magnetic field applied through a pair of RF coils. Finally, a photodetector was used to detect the resonance behavior of transmitted light intensity while the RF frequency was swept around the Larmor frequency. The photodetector output was connected to a lock-in amplifier for extraction of in-phase and quadrature components of the resonance signal.

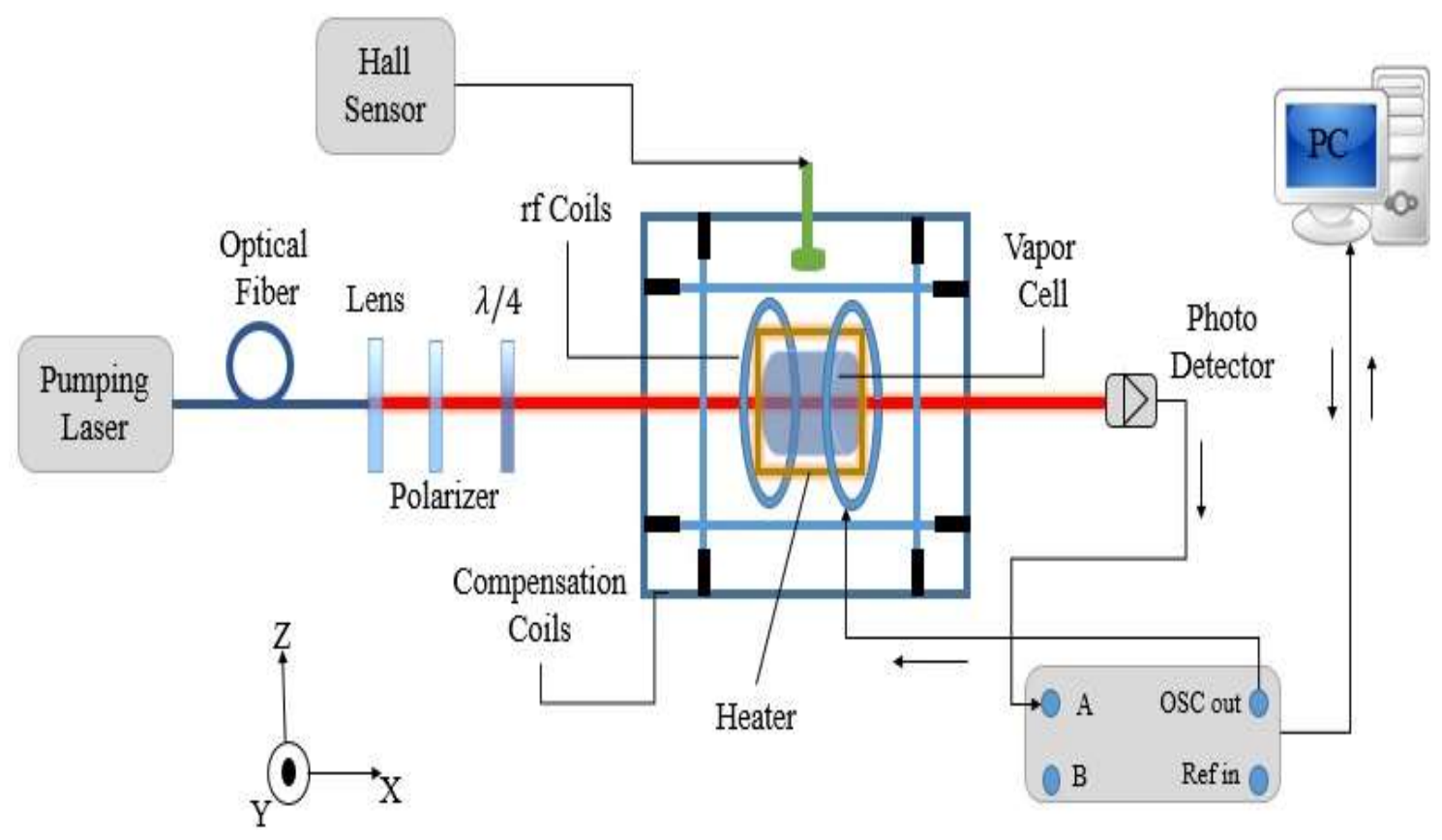

Fig. 1. Earth's magnetic field measurement setup. Laser beam was transported by an optical fiber to the magnetometer, where it was collimated and circularly polarized. Then, it passed the cell which was heated by a nonmagnetic heater, and finally the beam entered a photo-detector. The output of the photo-detector was fed to a lock-in amplifier and the lock-in controlled the frequency of RF coils. At the same time the output of photo-detector was read by a computer for data analysis. 
For compensation of the Earth's magnetic field, three pairs of square Helmholtz coils were used to generate the magnetic field along the axis of the coil-pair. In the first step of the compensation, the components of the Earth's magnetic field vector were estimated by using a Hall Effect sensor and were applied in opposite direction. At the next step, the residual magnetic field was compensated by zero field finding algorithm. The magnetic fields produced by the Helmholtz coils finally, were equivalent to the Earth's magnetic components.

\section{III.RESULTS AND DISCUSSIONS}

To create a homogeneous magnetic field in the range of the Earth's magnetic field and also to decrease the magnetic field gradient along the length of the cell, three pairs of square Helmholtz coils were designed (as shown in Fig. 2(a)). The coil side lengths were chosen to be 56,58 , and $60 \mathrm{~cm}$, with $\mathrm{N}=20$ number of turns.

The homogeneity of a magnetic field $(\mathrm{H})$ is a measure of its variability in a determined area of space. The magnetic field distribution generated by a Helmholtz configuration is homogenous only for a given area at half the distance separating the pairs of coils [13]. The homogeneity of the designed coils follows the shape in Fig. 2(b).

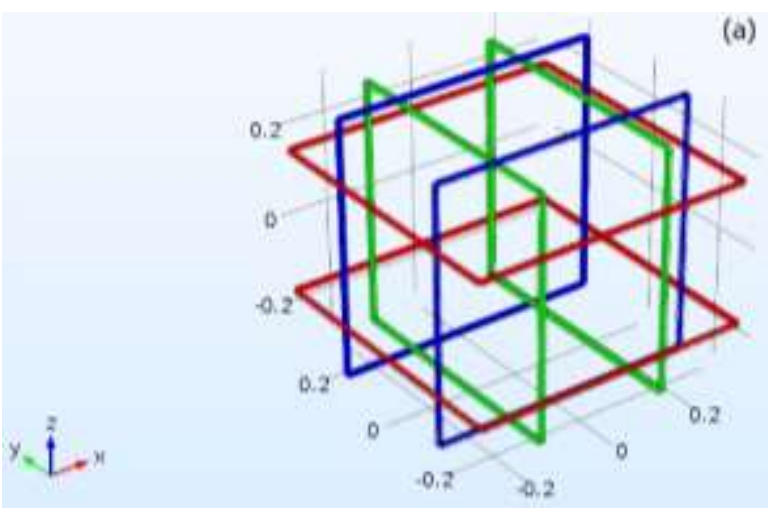

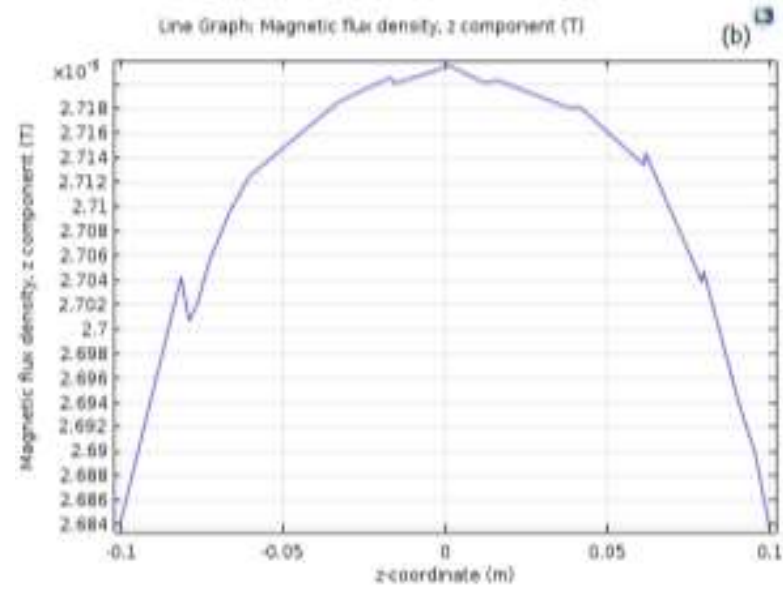

Fig. 2. Three pairs of Helmholtz coils simulation (a) and magnetic field distribution around the vapor cell produced by one pair (b)

The value of homogeneity in the cell length is \pm 0.098 by using the Eq. Error! Reference source not found.).

$$
H[\%]=\frac{B_{i}-B_{0}}{B_{0}} \times 100
$$

In Eq. Error! Reference source not found.), $B_{0}$ is the magnetic field at the center of square Helmholtz coils and $B_{i}$ is the magnetic field at another point within the cell length. The most common values of the magnetic field homogeneity are $\pm 0.1 \%, \pm 0.5 \%$ and $\pm 1 \%$. So, the value obtained for the designed coils is sufficient for the precise Earth's field measurement.

Fig. 3 shows the in-phase $(\mathrm{X})$ and quadrature (Y) components of the resonance signal while the Earth's magnetic field is approximately compensated by a Hall sensor. The green point indicates the Larmor frequency of $8.80 \pm 0.09$ $\mathrm{kHz}$, corresponding to the residual magnetic field of $1.913 \mu \mathrm{T}$, since $\omega=\gamma B$. In this equation $\omega$ is the resonance frequency (Larmor Frequency), $B$ is the magnetic field strength and $\gamma$ is the gyromagnetic ratio which is $0.46 \times 10^{10} \mathrm{~Hz} / \mathrm{T}$. 


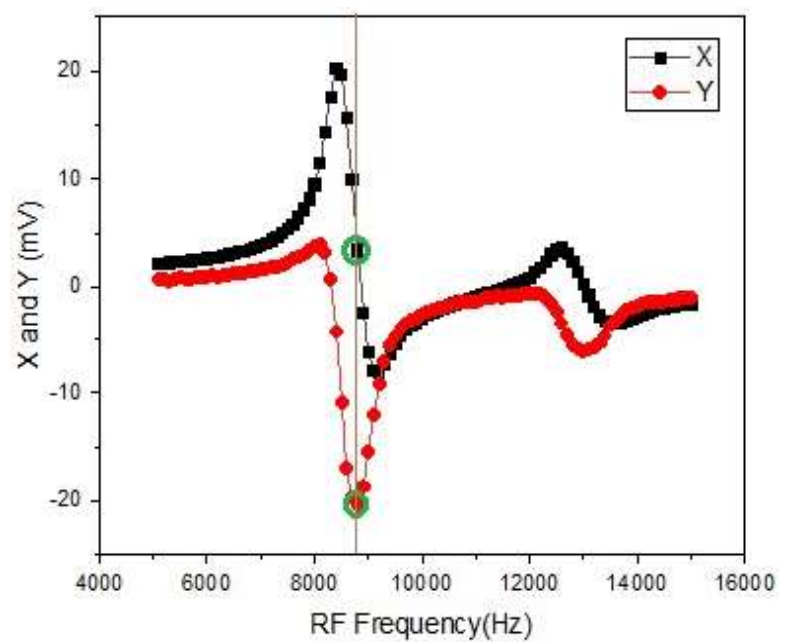

Fig. 3. In-phase and quadrature components of resonance signal. Green point shows the resonance frequency (i.e. residual magnetic field).

The residual magnetic field was decreased to its minimum value by using zero field finding algorithm. For this purpose, the approximate values of the Earth's magnetic field, obtained from the first step, were generated by the coils. Then the frequency of RF field was swept to obtain the resonance frequency. After that, a small magnetic field $\mathrm{dB}$ was applied via one of the three coil pairs. The RF frequency was swept again and the obtained resonance frequencies from both cases were compared to each other. If the second value was less than the first one, then $\mathrm{dB}$ was set to $2 \mathrm{~dB}$, and the process was repeated. If it was more than the first one, $\mathrm{dB}$ was set to $\mathrm{dB} / 2$, and applied in the opposite direction and the process was repeated again. Finally, the minimum possible value of resonance frequency (corresponding to minimum magnetic field) was achieved.

This process was performed for one axis. The same process was executed for two other axes. As the acquired components compensate the Earth magnetic field vector to zero, they can be regarded as the measured value of the fields with opposite sign.

Fig. 4 shows the variation of Larmor frequency versus the magnetic field in $y$ direction. The current applied to the coil pairs was converted to magnetic field strength by

$$
B_{y}(0)=\frac{4 \mu_{0} N I}{1.2965 \pi \sqrt{2.2965 a}}
$$

where $N$ is the number of turns, $I$ is the applied current, and a is half side length of square Helmholtz coils [13].

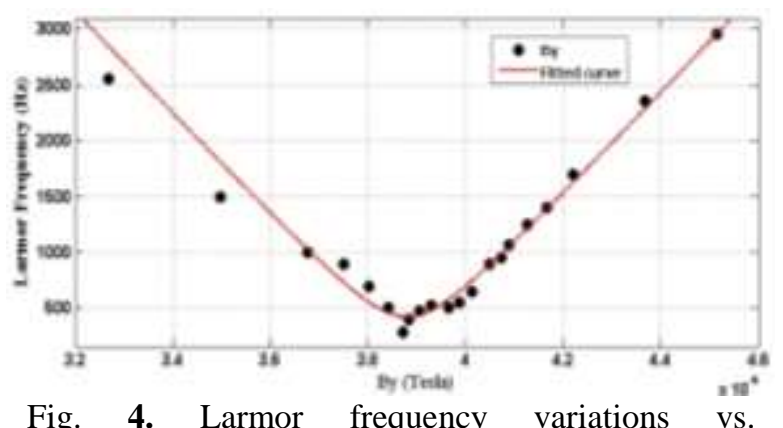
compensating magnetic field in $y$ direction.

To find the minimum magnetic field in each direction, the function

$\gamma \sqrt{a^{2}+\left(B_{y}-b\right)^{2}}$

fitted the experimental data, since $a^{2}=B_{x}^{2}+B_{z}^{2} \quad$ (as shown in Fig. 4). The parameter $b$, obtained as a result of fitting, has a value of $3.890 \mu \mathrm{T}$ and shows the Earth's magnetic field in y direction. In the same way, $\mathrm{x}$ and $\mathrm{z}$ components of Earth's magnetic field are calculated to be $15.433 \mu \mathrm{T}$ and $31.320 \mu \mathrm{T}$ on the location of measurement point. As the resonance frequency is achieved by resolution of $10 \mathrm{~Hz}$, the Earth's magnetic field accuracy is about $2 \mathrm{nT}$.

\section{IV.CONCLUSION}

High sensitivity atomic magnetometers play a significant role in precise measurement of the Earth's magnetic field vector. However, these magnetometers have to operate in an unshielded environment. Therefore, in this paper, we have proposed a method based on zero field finding algorithm in an unshielded environment. According to this method, the Earth's field components were measured and applied oppositely so the magnetic field in the center was compensated. Then, small portions of magnetic field were applied to the Helmholtz coils until the minimum residual 
field was achieved. The applied field in each direction indicated the magnitude of the Earth's magnetic field vector. This value was calculated to be $35.132 \mu \mathrm{T}$ with $2 \mathrm{nT}$ accuracy in the lab.

\section{REFERENCES}

[1] C.J. Readings, The nine candidate Earth Explorer missions. 5. Magnetometry Mission, European Space Agency, Paris (France), 1996.

[2] A. Meloni and R. Lanza, The Earths magnetism, an introduction for geologist, Springer, 2008.

[3] R. Čop and D. Fefer, Nature of Earth's magnetic field and its application for commercial flight navigation, Geomagnetics for Aeronautical Safety, Springer, Dordrecht, 2006.

[4] M.J. Caruso, "Applications of magnetoresistive sensors in navigation systems," SAE Technical Paper, Vol. 1220, pp. 15-21, 1997.

[5] W. Goesta, D.B. Ericson, W.B. Ryan, and J.H. Foster, "Magnetism of the Earth and climatic changes," Earth Planet. Sci. Lett. Vol. 12, pp. 175-183, 1971.

[6] K. Korth, K. Strohbehn, F. Tejada, A.G. Andreou, J. Kitching, S. Knappe, S.J. Lehtonen, S.M. London, and M. Kafel, "Miniature atomic scalar magnetometer for space based on the rubidium isotope $87 \mathrm{Rb}$," J. Geophys. Res: Space Phys. Vol. 121, pp. 7870-7880, 2016.

[7] T. Schönau, M. Schmelz, V. Zakosarenko, R. Stolz, M. Meyer, S. Anders, L. Fritzsch, and H.-G. Meyer, "SQUID-based setup for the absolute measurement of the Earth's magnetic field," Supercond. Sci. Technol. Vol. 26, pp. 035013 (1-8), 2013.

[8] J.M. Leger, F. Bertrand, T. Jager, M. L. Prado, I. Fratter, and J. C. Lalaurie, "Swarm absolute scalar and vector magnetometer based on helium 4 optical pumping," Procedia Chem. Vol. 1, pp. 634-637, 2009.

[9] S. J.Seltzer and M.V. Romalis, "Unshielded three-axis vector operation of a spinexchange-relaxation-free atomic magnetometer," Appl. Phys. Lett. Vol. 85 (20), pp. 4804-4806, 2004.
[10]M. Ranjbaran, M.M. Tehranchi, S.M. Hamidi, and S.M.H. Khalkhali, "Harmonic detection of magnetic resonance for sensitivity improvement of optical atomic magnetometers," J. Magn. Magn. Mater. Vol. 424, pp. 284-290, 2017.

[11]J.M. Leger, F. Bertrand, T. Jager, M.L. Prado, I. Fratter, and J.C. Lalaurie, "Swarm absolute scalar and vector magnetometer based on helium 4 optical pumping," Procedia Chem. Vol. 1, pp. 634-637, 2009.

[12]H. Dong, H. Lin, and X. Tang, "Atomicsignal-based zero-field finding technique for unshielded atomic vector magnetometer," IEEE Sensors J. Vol. 13, pp. 186-189, 2013.

[13]A. Restrepo, F. Andres, F.M. Edinson, and R. P.J. Carlos, "Study and analysis of magnetic field homogeneity of square and circular Helmholtz coil pairs: A Taylor series approximation," VI Andean Region International Conference Andescon, Cuenca, Vol. 27, pp. 77-80, 2012.

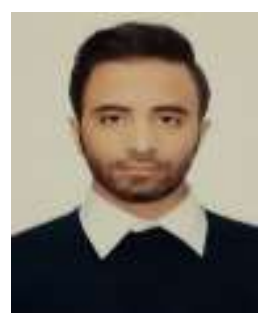

Amin Zamani recieved his B.Sc. in optics and laser engineering from Shahid Bahonar University, Iran, in 2016 and M.S. in photonics from Shahid Beheshti University in 2019. He is currently working at MagnetoPhotonic Lab, Shahid Beheshti University, Iran. His research interests includes optics and laser and their applications in magnetometry. 


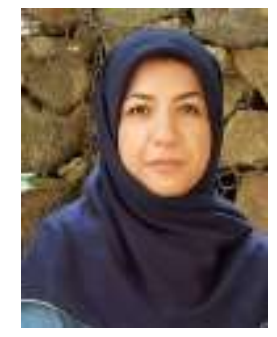

Maliheh Ranjbaran received her M.S. and $\mathrm{Ph}$. D. in photonics from Laser and Plasma Research institute, Shahid Beheshti University, Iran, in 2010 and 2018, respectively. She is currently a post-doctoral researcher at this University. Her research interest includes optical measurement systems, magnetic sensors and their applications.

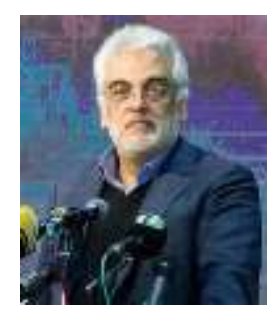

Mohammad Mehdi Tehranchi received the $\mathrm{Ph}$. D. degree in physics from Prokhorov General Physics Institute of the Russian Academy of Sciences (GPI RAS) in 1997. He is currently a professor of physics and the director of magneto-photonic lab of laser and plasma research institute and Physics department of Shahid Beheshti University. He has worked on the research fields of magnetic materials (such as amorphous materials, multiferroic materials and magnetophotonic crystals) and magnetic effects (such as linear and nonlinear magneto-optical effects and
Giant magnetoimpedance effects) which are utilized in magnetic sensors and nondestructive testing technology.

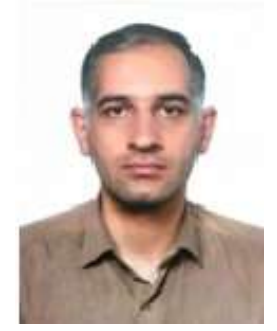

Seyed Mohammad Hosein Khalkhali, received the $\mathrm{Ph}$. D. degree in physics from physics department of Shahid Beheshti University, Iran, in 2014. He has worked on the ferroelectric and photovoltaic composite thin films.

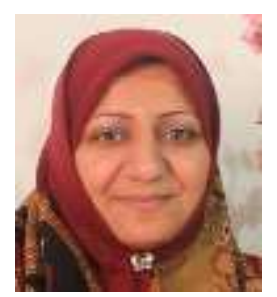

Seyedeh Mehri Hamidi recieved her Ph.D. degree in photonics from Laser and Plasma Research Institute, Shahid Beheshti University, Tehran, Iran, in 2009. She is currently the director of magneto-plasmonic lab of Laser and Plasma Research Institute. She has worked on the research fields of magneto-plasmonic, nanophotonics, photonic and magneto phtotonic crystals, surface plasmon resonance, dielectric and magnetic waveguides and pulsed laser deposition technique. 